\title{
A UNICITY THEOREM FOR MOVING TARGETS COUNTING MULTIPLICITIES
}

\author{
LU JIN AND MIN RU
}

(Received December 8, 2003, revised July 27, 2004)

\begin{abstract}
R. Nevanlinna showed, in 1926, that for two nonconstant meromorphic functions on the complex plane, if they have the same inverse images counting multiplicities for four distinct complex values, then they coincide up to a Möbius transformation, and if they have the same inverse images counting multiplicities for five distinct complex values, then they are identical. H. Fujimoto, in 1975, extended Nevanlinna's result to nondegenerate holomorphic curves. This paper extends Fujimoto's uniqueness theorem to the case of moving hyperplanes in pointwise general position.
\end{abstract}

1. Introduction. In Nevanlinna theory, there is a well-known uniqueness theorem due to R. Nevanlinna. He showed, in 1926, that any two meromorphic functions sharing four distinct values must coincide up to a Möbius transformation, and if they share five distinct values, then they must be identical. In 1975, H. Fujimoto (see [F]) extended this result to holomorphic curves, and proved the following.

THEOREM A (Fujimoto $[\mathrm{F}])$. Let $f$ and $g$ be two non-constant holomorphic curves of the complex plane $\boldsymbol{C}$ into the complex projective $n$-space $\boldsymbol{P}^{n}(\boldsymbol{C})$. Suppose that there exist $3 n+1$ hyperplanes $H_{j}, 1 \leq j \leq 3 n+1$, in $\boldsymbol{P}^{n}(\boldsymbol{C})$ located in general position such that $f(\boldsymbol{C}) \not \subset H_{j}, g(\boldsymbol{C}) \not \subset H_{j}$ and $v\left(f, H_{j}\right)=v\left(g, H_{j}\right), 1 \leq j \leq 3 n+1$, where $v\left(f, H_{j}\right)$ and $v\left(g, H_{j}\right)$ denote the pullbacks of the divisors $\left(H_{j}\right)$ by $f$ and $g$, respectively. Then there is a projective linear transformation $L$ of $\boldsymbol{P}^{n}(\boldsymbol{C})$ such that $L(f)=g$.

THEOREM B (Fujimoto [F]). Let $f$ and $g$ be two non-constant holomorphic curves of $\boldsymbol{C}$ into $\boldsymbol{P}^{n}(\boldsymbol{C})$, at least one of which is non-degenerate. Suppose that there exist $3 n+2$ hyperplanes $H_{j}, 1 \leq j \leq 3 n+2$, in $\boldsymbol{P}^{n}(\boldsymbol{C})$ located in general position such that $f(\boldsymbol{C}) \not \subset H_{j}$, $g(\boldsymbol{C}) \not \subset H_{j}$ and $v\left(f, H_{j}\right)=v\left(g, H_{j}\right), 1 \leq j \leq 3 n+2$, where $v\left(f, H_{j}\right)$ and $v\left(g, H_{j}\right)$ denote the pullbacks of the divisors $\left(H_{j}\right)$ by $f$ and $g$, respectively. Then $f=g$.

Recently, there appeared two papers (see [Ye] and [Tu]) which extend the above results to moving targets. However, both papers imposed additional conditions (see the condition (ii) and the condition (iii) in Theorem $\mathrm{C}$ below). In particular, the condition of $f$ and $g$ being equal on $f^{-1}\left(H_{j}\right), 1 \leq j \leq q$, (see condition (iii) in Theorem $\mathrm{C}$ below) seems unnatural. Furthermore, as indicated in [Tu], the paper of [Ye] contains some gaps in his key argument.

2000 Mathematics Subject Classification. Primary 32H30; Secondary 32H25.

Key words and phrases. Holomorphic maps, unicity theorem, moving targets.

The first author is supported by National Natural Science Foundation of China (No. 10271029). The second author is supported in part by NSA under grant number MSPF-02G-175. 
To state the results obtained in [Tu], we first introduce some notation. Throughout this paper, we denote by $\mathbf{f}$ a reduced representation of a holomorphic map $f: \boldsymbol{C} \rightarrow \boldsymbol{P}^{n}(\boldsymbol{C})$. By a moving hyperplane $H$ in $\boldsymbol{P}^{n}(\boldsymbol{C})$, we mean

$$
H=\left\{\left[x_{0}: \cdots: x_{n}\right] \mid \sum_{i=0}^{n} a_{i} x_{i}=0\right\},
$$

where $a_{0}, \ldots, a_{n}$ are entire functions without common zeros. So $H$ is associated with a holomorphic map $a=\left[a_{0}: \cdots: a_{n}\right]: \boldsymbol{C} \rightarrow \boldsymbol{P}^{n}(\boldsymbol{C})$. Write $\mathbf{a}=\left(a_{0}, \ldots, a_{n}\right)$. Given a holomorphic map $f: \boldsymbol{C} \rightarrow \boldsymbol{P}^{n}(\boldsymbol{C})$, we say that $f$ and $H$ (or $f$ and $a$ ) are free if $\langle\mathbf{f}, \mathbf{a}\rangle \not \equiv 0$, where $\mathbf{f}$ is a reduced representation of $f$ and $\langle$,$\rangle is the standard inner product on \boldsymbol{C}^{n+1}$. The moving hyperplanes $H_{1}, \ldots, H_{q}$ (or $\mathbf{a}_{1}, \ldots, \mathbf{a}_{q}$ ) are said to be in general position if $H_{1}(z), \ldots, H_{q}(z)$ are in general position for some (and hence for almost all) $z \in \boldsymbol{C}$. The main result obtained in $[\mathrm{Tu}]$ reads as follows.

THEOREM C (Tu [Tu]). Let $f$ and $g$ be two non-constant holomorphic curves of $\boldsymbol{C}$ into $\boldsymbol{P}^{n}(\boldsymbol{C})$. Let $\mathcal{G}=\left\{H_{j}\right\}_{j=1}^{q}\left(\right.$ or $\left.\left\{a_{j}\right\}_{j=1}^{q}\right)$ be a finite set of moving hyperplanes in $\boldsymbol{P}^{n}(\boldsymbol{C})$, located in general position. Let $\mathbf{a}_{j}=\left(a_{j, 0}, \ldots, a_{j, n}\right)$. Let $\mathcal{R}_{\mathcal{G}}$ be the smallest field containing $C$ and all $a_{j \mu} / a_{j v}$ with $a_{j v} \not \equiv 0$, and let $\tilde{\mathcal{R}}_{\mathcal{G}}$ be the smallest field containing all meromorphic functions $h$ such that $h^{k} \in \mathcal{R}_{\mathcal{G}}$. Assume that $\max _{1 \leq j \leq q} T_{a_{j}}(r)=o\left(T_{f}(r)\right)$ and $f$ is linearly nondegenerate over $\mathcal{R}_{\mathcal{G}}$. Assume further that

(i) $\left\langle\mathbf{f}, \mathbf{a}_{j}\right\rangle /\left\langle\mathbf{g}, \mathbf{a}_{j}\right\rangle, 1 \leq j \leq q$, are nowhere zero entire functions on $\boldsymbol{C}$,

(ii) $\left\{z \mid\left\langle\mathbf{f}(z), \mathbf{a}_{i}(z)\right\rangle=0\right\} \cap\left\{z \mid\left\langle\mathbf{f}(z), \mathbf{a}_{j}(z)\right\rangle=0\right\}=\emptyset$ for $1 \leq i<j \leq q$,

(iii) $f=g$ on $\bigcup_{j=1}^{q}\left\{z \in \boldsymbol{C} \mid\left\langle\mathbf{f}(z), \mathbf{a}_{j}(z)\right\rangle=0\right\}$.

Then the following hold:

(a) If $q=3 n+1$, then there exists an $(n+1) \times(n+1)$ matrix $L$ with entries in $\tilde{\mathcal{R}}_{\mathcal{G}}$ and $\operatorname{det}(L) \not \equiv 0$ such that $\mathbf{f}=L \cdot \mathbf{g}$, where $\mathbf{f}$ (resp. $\mathbf{g}$ ) is a reduced representation of $f$ (resp. $g$ ).

(b) If $q=3 n+2$, and, in addition, if $f$ is linearly nondegenerate over $\tilde{\mathcal{R}}_{\mathcal{G}}$, then $f=g$.

The purpose of this paper is to extend Fujimoto's results (Theorem A and Theorem B) to moving hyperplanes without conditions (ii) and (iii) assumed in Theorem C. Also, we do not assume that the hyperplanes are slowly moving with respect to the growth of $f$, i.e., we do not assume that $\max _{1 \leq j \leq q} T_{a_{j}}(r)=o\left(T_{f}(r)\right)$. Instead, we assume that the given moving hyperplanes are located in pointwise general position. Recall that moving hyperplanes $H_{j}, 1 \leq j \leq q$, are said to be in pointwise general position (vs. in general position) if the hyperplanes $H_{j}(z), 1 \leq j \leq q$, are in general position (as a set of fixed hyperplanes) at every point $z \in \boldsymbol{C}$ (vs. some point $z_{0} \in \boldsymbol{C}$ ). With this assumption, we prove the following result.

MAIN THEOREM. Let $\mathcal{G}=\left\{H_{j}\right\}_{j=1}^{q}\left(\right.$ or $\left.\left\{a_{j}\right\}_{j=1}^{q}\right)$ be a finite set of moving hyperplanes in $\boldsymbol{P}^{n}(\boldsymbol{C})$, located in pointwise general position. Let $f$ and $g$ be two non-constant holomorphic mappings of $\boldsymbol{C}$ into $\boldsymbol{P}^{n}(\boldsymbol{C})$, such that $\left\langle\mathbf{f}, \mathbf{a}_{j}\right\rangle \not \equiv 0$ and $\left\langle\mathbf{g}, \mathbf{a}_{j}\right\rangle \not \equiv 0$ for $1 \leq j \leq q$. Assume that $\left\langle\mathbf{f}, \mathbf{a}_{j}\right\rangle /\left\langle\mathbf{g}, \mathbf{a}_{j}\right\rangle, 1 \leq j \leq q$, are nowhere zero entire functions on $\boldsymbol{C}$. Then the following hold: 
(a) If $q=3 n+1$, then there exists an $(n+1) \times(n+1)$ invertible matrix $L$ with entire functions as entries, such that $\mathbf{f}=L \cdot \mathbf{g}$, where $\mathbf{f}$ (resp. $\mathbf{g}$ ) is a reduced representation of $f$ (resp. $g$ ). In addition, the entries of $L$ depend only on the given hyperplanes and can be determined effectively.

(b) If $q=3 n+2$, and, in addition, if $f$ is linearly nondegenerate over $\tilde{\mathcal{R}}_{\mathcal{G}}$, then $f=g$. Here $\mathcal{R}_{\mathcal{G}}$ is the smallest field containing $\boldsymbol{C}$ and all $a_{j \mu} / a_{j v}$ with $a_{j v} \not \equiv 0$, and $\tilde{\mathcal{R}}_{\mathcal{G}}$ is the smallest field containing all meromorphic functions $h$ such that $h^{k} \in \mathcal{R}_{\mathcal{G}}$.

Note that the main theorem can be extended easily to holomorphic maps $f$ and $g$ from $\boldsymbol{C}^{m}$ to $\boldsymbol{P}^{n}$

The second author wishes to thank the Department of Mathematics, Fudan University, P.R. China for kind hospitality and support, during which part of the work on this paper took place.

2. Proof of the main theorem. To prove the main theorem, we recall the following Borel's lemma (see $[\mathrm{Ru}]$ or $[\mathrm{L}])$.

BOREL's LEMMA. Let $f_{0}, \ldots, f_{n+1}$ be nowhere zero entire functions such that

$$
f_{0}+\cdots+f_{n+1}=0 .
$$

Let $\{0,1, \ldots, n+1\}=I_{1} \cup I_{2} \cdots \cup I_{k}$ be the partition such that $i$ and $j$ are in the same class $I_{l}$ if and only if $f_{i}=c_{i j} f_{j}$ for some nonzero constant $c_{i j}$. Then

$$
\sum_{i \in I_{l}} f_{i}=0
$$

for any $l$.

Consider the moving hyperplanes

$$
H_{j}=\left\{\left[x_{0}: \cdots: x_{n}\right] \mid a_{j 0} x_{0}+\cdots+a_{j n} x_{n}=0\right\}, \quad 1 \leq j \leq q,
$$

where $a_{j i}, 1 \leq j \leq q, 0 \leq i \leq n$, are entire functions without common zeros. Write $\mathbf{a}_{j}=\left(a_{j 0}, \ldots, a_{j n}\right)$, and let $\mathbf{f}$ be a reduced representation of $f$. We first prove the part (a) in the main theorem. We assume that $q \geq 3 n+1$ in this case. Define functions

$$
h_{j}:=\left\langle\mathbf{f}, \mathbf{a}_{j}\right\rangle /\left\langle\mathbf{g}, \mathbf{a}_{j}\right\rangle, \quad 1 \leq j \leq q .
$$

Then, by the assumption, $h_{j}$ are nowhere zero entire functions. We need the following two claims. We note that, although the situation is different, the proofs of Claim 1, Claim 2 and the Combinatorial Lemma below follow the argument given by Fujimoto (see [F], or [F2]). We enclose the proofs in this paper for the sake of reader's convenience.

Claim 1. Let $T$ be any subset of $\{1,2, \ldots, q\}$ with $\# T=2 n+2$, where $q \geq 3 n+1$. Then, for each $I \subset T$ with $\# I=n+1$, there exists a set $J \subset T$ with $\# J=n+1, I \neq J$, such that $h_{I} / h_{J}$ is a nowhere zero entire function depending only on the given hyperplanes and can be determined effectively, where $h_{I}:=h_{i_{0}} \ldots h_{i_{n}}$ for $I=\left\{i_{0}, \ldots, i_{n}\right\}$. 
To prove this claim, we assume without loss of generality that $T=\{1,2, \ldots, 2 n+2\}$. Write (2.1) as

$$
a_{j 0} f_{0}+\cdots+a_{j n} f_{n}=h_{j}\left(a_{j 0} g_{0}+\cdots+a_{j n} g_{n}\right), \quad j \in T .
$$

By Cramer's rule for solving a system of linear equations, we obtain

$$
\operatorname{det}\left(a_{j 0}, \ldots, a_{j n}, a_{j 0} h_{j}, \ldots, a_{j n} h_{j} ; j=1,2, \ldots, 2 n+2\right)=0 .
$$

Then, by the Laplace expansion theorem,

$$
\sum_{J \subset T, \# J=n+1} \alpha_{J} A_{J} h_{J}=0,
$$

where $J=\left\{i_{0}, \ldots, i_{n}\right\}, \alpha_{J}=(-1)^{n(n+1) / 2+i_{0}+\cdots+i_{n}}$, and

$$
A_{J}=\operatorname{det}\left(a_{i_{r}, j}\right)_{0 \leq r \leq n, 0 \leq j \leq n} \operatorname{det}\left(a_{i_{s}^{\prime}, j}\right)_{0 \leq s \leq n, 0 \leq j \leq n}
$$

for $i_{0}^{\prime}, \ldots, i_{n}^{\prime} \in T-J$. Since $H_{1}, \ldots, H_{q}$ are in pointwise general position, any $n+1$ vectors in $\left\{\left(a_{j 0}, \ldots, a_{j n}\right) \mid 1 \leq j \leq 2 n+2\right\}$ are linearly independent at each point $z \in C$. Therefore, $A_{J}$ is nowhere zero for all $J$ 's. Hence, by applying Borel's lemma, there exists constant $c_{I J}$ and $J \subset T, J \neq I$ such that $A_{I} h_{I}=c_{I J} A_{J} h_{J}$, and hence $h_{I} / h_{J}=c_{I J} A_{J} / A_{I}$. So we obtain Claim 1.

Our next step is to prove the following claim.

Claim 2. Let $q \geq 3 n+1$. There is a subset $I_{0}$ of $\{1,2, \ldots, q\}$ with $\# I_{0}=q-2 n$ such that $h_{i} / h_{j}$, for every $i, j \in I_{0}$, is a nowhere zero entire function depending only on the given hyperplanes and can be determined effectively.

To prove Claim 2, consider the multiplicative group $\mathcal{H}^{*}$ of all nowhere zero entire functions. Denote by $\mathcal{T}$ the smallest subgroup of $\mathcal{H}^{*}$ which contains all $h \in \mathcal{H}^{*}$ with $h^{k} \in \mathcal{R}_{\mathcal{G}}$ for some positive integer $k$. So we have $\mathcal{H}^{*} \cap \mathcal{R}_{\mathcal{G}} \subset \mathcal{T} \subset \tilde{\mathcal{R}}_{\mathcal{G}}$. Then the multiplication group $\mathcal{H}^{*} / \mathcal{T}$ is a torsion free abelian group. We denote by $[h]$ the class in $\mathcal{H}^{*} / \mathcal{T}$ containing $h \in \mathcal{H}^{*}$. Consider the subgroup $\widetilde{\mathcal{G}}$ of $\mathcal{H}^{*} / \mathcal{T}$ generated by $\left[h_{1}\right], \ldots,\left[h_{q}\right]$ and choose suitable functions $b_{1}, \ldots, b_{t} \in \mathcal{H}^{*}$ such that $\left[b_{1}\right], \ldots,\left[b_{t}\right] \in \mathcal{H}^{*} / \mathcal{T}$ give a basis of $\widetilde{\mathcal{G}}$. Then each $h_{j}$ can be uniquely represented as

$$
h_{j}=g_{j} b_{1}^{r_{j 1}} \cdots b_{t}^{r_{j t}}, \quad 1 \leq j \leq q,
$$

with some $g_{j} \in \mathcal{T}$ and integers $r_{j \tau}, 1 \leq \tau \leq t$.

To proceed, we need the following combinatorial lemma.

COMbinatorial Lemma. Given integers $r_{j \tau}, 1 \leq j \leq q, 1 \leq \tau \leq t$, we can choose integers $\beta_{1}, \ldots, \beta_{t}$ such that among the integers

$$
r_{j}:=r_{j 1} \beta_{1}+\cdots+r_{j t} \beta_{t}, \quad 1 \leq j \leq q,
$$

any two numbers, say, $r_{i}$ and $r_{j}$ are equal only if the corresponding vectors $\left(r_{i 1}, \ldots, r_{i t}\right)$ and $\left(r_{j 1}, \ldots, r_{j t}\right)$ are equal. 
The Combinatorial Lemma is the same as that in [F] page 2, (2.2), or [F2] page 118, (3.4.13). We enclose the proof here again for the sake of reader's convenience. The proof is given by induction. In fact, the lemma is trivial for the case $t=1$. Assume that there exist $\beta_{1}, \ldots, \beta_{t-1}$ with the property that if $r_{i}^{*}=r_{j}^{*}$ for integers $r_{i}^{*}:=r_{i 1} \beta_{1}+\cdots+r_{i, t-1} \beta_{t-1}$, then $\left(r_{i 1}, \ldots, r_{i, t-1}\right)=\left(r_{j 1}, \ldots, r_{j, t-1}\right)$. Then it is easy to show that there are only finitely many integers $\beta_{t}$ such that $\beta_{1}, \beta_{2}, \ldots, \beta_{t}$ do not satisfy the desired condition. Thus the lemma is proved.

We now prove Claim 2. Let $r_{j \tau}, 1 \leq j \leq q, 1 \leq \tau \leq t$, be the integers given by (2.6), and choose $\beta_{1}, \ldots, \beta_{t}$ as in the Combinatorial Lemma. Define $r_{j}, 1 \leq j \leq q$, by (2.7). After a suitable change of indices, we may assume that

$$
r_{1} \leq \cdots \leq r_{q} .
$$

If $r_{n+1}=\cdots=r_{q-n}$, then $r_{n+1, \tau}=\cdots=r_{q-n, \tau}$ for $1 \leq \tau \leq t$. So by (2.6), $h_{i} / h_{j}=$ $g_{i} / g_{j} \in \mathcal{T}$ for $i, j \in\{n+1, \ldots, q-n\}$. Hence the proof of Claim 2 would be finished if we can show that $r_{n+1}=\cdots=r_{q-n}$. To prove this, take $T=\{1, \ldots, n+1, q-n, \ldots, q\}$ which contains $2 n+2$ elements. Applying Claim 1 with $I=\{1,2, \ldots, n+1\}$, we see that there is a subset $J=\left\{i_{0}, \ldots, i_{n}\right\}$ of $T$ such that $\left\{i_{0}, \ldots, i_{n}\right\} \neq\{1,2, \ldots, n+1\}$ and

$$
\frac{h_{i_{0}} h_{i_{1}} \cdots h_{i_{n}}}{h_{1} h_{2} \cdots h_{n+1}} \in \mathcal{T} \text {. }
$$

From (2.6) this implies that $b_{1}^{l_{1}} \cdots b_{t}^{l_{t}}=g b_{1}^{l_{1}^{\prime}} \cdots b_{t}^{l_{t}^{\prime}}$, where $l_{\tau}=r_{i_{0} \tau}+\cdots+r_{i_{n} \tau}, l_{\tau}^{\prime}=$ $r_{1 \tau}+\cdots+r_{n+1, \tau}, 1 \leq \tau \leq t$, and $g \in \mathcal{T}$. Since $b_{1}, \ldots, b_{t}$ are multiplicatively independent, we have $l_{\tau}=l_{\tau}^{\prime}$ for $\tau=1,2, \ldots, t$, that is,

$$
r_{i_{0} \tau}+\cdots+r_{i_{n} \tau}=r_{1 \tau}+\cdots+r_{n+1 \tau} .
$$

Thus we obtain

$$
\sum_{s=0}^{n} r_{i_{s}}=\sum_{s=0}^{n} \sum_{\tau=1}^{t} r_{i_{s} \tau} \beta_{\tau}=\sum_{i=1}^{n+1} \sum_{\tau=1}^{t} r_{i \tau} \beta_{\tau}=\sum_{i=1}^{n+1} r_{i}
$$

which simply means that

$$
\left(r_{i_{0}}-r_{1}\right)+\cdots+\left(r_{i_{n}}-r_{n+1}\right)=0 .
$$

Since $r_{i_{0}} \geq r_{1}, \ldots, r_{i_{n}} \geq r_{n+1}$, this is possible only when $r_{i_{n}}=r_{n+1}$. However, since $i_{n} \geq q-n$, by (2.8) we have $r_{i_{n}} \geq r_{q-n}$. Thus $r_{n+1} \geq r_{q-n}$. Noticing that $r_{n+1} \leq r_{q-n}$, we have $r_{n+1}=r_{q-n}$. Using (2.8) again, we obtain that $r_{n+1}=\cdots=r_{q-n}$. This concludes Claim 2.

We now prove the part (a). Again we follow Fujimoto's argument (see [F] or [F2]). By Claim 2 with $q=3 n+1$ together with a suitable change of the reduced representation, we may assume, without loss of generality, that $h_{1}, \ldots, h_{n+1}$ are the entire functions which depend only on the given hyperplanes, and can be determined effectively. We define $A:=$ $\left(a_{j i}\right)_{1 \leq j \leq n+1,0 \leq i \leq n}$ and $H$ to be a diagonal matrix with diagonal entries as $h_{1}, \ldots, h_{n+1}$. Then, by (2.1), $A \mathbf{f}=H A \mathbf{g}$. Hence $\mathbf{f}=A^{-1} H A \mathbf{g}$, and the entries of the matrix $A^{-1} H A$ 
depend only on the given hyperplanes and can be determined effectively. Hence the part (a) in the Main Theorem is proved.

To prove the part (b), since $q=3 n+2$, by Claim 2 together with a suitable change of the reduced representation, we may assume, without loss of generality, that $h_{1}, \ldots, h_{n+2}$ are the entire functions which depend only on the given hyperplanes, and can be determined effectively. Again, let $A:=\left(a_{j i}\right)_{1 \leq j \leq n+1,0 \leq i \leq n}$. Then, by (2.1), $A \mathbf{f}=H A \mathbf{g}$, where $H$ is a diagonal matrix with diagonal entries as $h_{1}, \ldots, h_{n+1}$, and

$$
\left(a_{n+2,0}, \ldots, a_{n+2, n}\right)\left(\begin{array}{c}
f_{0} \\
f_{1} \\
\vdots \\
f_{n}
\end{array}\right)=h_{n+2}\left(a_{n+2,0}, \ldots, a_{n+2, n}\right)\left(\begin{array}{c}
g_{0} \\
g_{1} \\
\vdots \\
g_{n}
\end{array}\right) \text {. }
$$

Hence

$$
\left(a_{n+2,0}, \ldots, a_{n+2, n}\right)\left(\begin{array}{c}
f_{0} \\
f_{1} \\
\vdots \\
f_{n}
\end{array}\right)=h_{n+2}\left(a_{n+2,0}, \ldots, a_{n+2, n}\right) A^{-1} H^{-1} A\left(\begin{array}{c}
f_{0} \\
f_{1} \\
\vdots \\
f_{n}
\end{array}\right) .
$$

Since $f$ is linearly nondegenerate over $\tilde{\mathcal{R}}_{\mathcal{G}}$, we deduce that $\left(a_{n+2,0}, \ldots, a_{n+2, n}\right)=$ $h_{n+2}\left(a_{n+2,0}, \ldots, a_{n+2, n}\right) A^{-1} H^{-1} A$. Therefore,

$$
\left(a_{n+2,0}, \ldots, a_{n+2, n}\right) A^{-1}\left(\begin{array}{cccc}
h_{1}-h_{n+2} & 0 & \cdots & 0 \\
0 & h_{2}-h_{n+2} & \cdots & 0 \\
\vdots & \vdots & \ddots & \vdots \\
0 & 0 & \cdots & h_{n+1}-h_{n+2}
\end{array}\right)=\mathbf{0} .
$$

Let

$$
\left(a_{n+2,0}, \ldots, a_{n+2, n}\right)=\left(b_{0}, \ldots, b_{n}\right) A .
$$

Since $\left\{H_{j}\right\}_{j=1}^{q}\left(\right.$ or $\left.\left\{a_{j}\right\}_{j=1}^{q}\right)$ are in pointwise general position, we have $b_{j} \not \equiv 0(j=0,1, \ldots, n)$, and

$$
\left(b_{0}, \ldots, b_{n}\right)\left(\begin{array}{cccc}
h_{1}-h_{n+2} & 0 & \cdots & 0 \\
0 & h_{2}-h_{n+2} & \cdots & 0 \\
\vdots & \vdots & \ddots & \vdots \\
0 & 0 & \cdots & h_{n+1}-h_{n+2}
\end{array}\right)=\mathbf{0}
$$

which implies that $h_{j}=h_{n+2}(j=1, \ldots, n+1)$. Hence from $A \mathbf{f}=H A \mathbf{g}=A H \mathbf{g}$, we deduce $\mathbf{f}=H \mathbf{g}$, which implies that $f=g$. The proof of the Main Theorem is completed.

\section{REFERENCES}

[F] H. Fujimoto, The uniqueness problem of meromorphic maps into the complex projective space, Nogoya Math. J. 58 (1975), 1-23. 
[F2] H. Fujimoto, Value distribution theory of the Gauss map of minimal surfaces in $\boldsymbol{R}^{m}$, Aspects Math. E21, Vieweg, Braunschweing, 1993.

[L] S. LANG, Introduction to complex hyperbolic spaces, Springer-Verlag, New York, 1987.

[Ru] M. RU, Nevanlinna theory and its relation to Diophantine approximation, World Scientific Publishing Co., Singapore, 2001.

[RS1] M. RU AND W. Stoll, The second main theorem for moving targets, J. Geom. Anal. 1 (1991), 99-138.

[RS2] M. RU AND W. Stoll, The Cartan conjecture for moving targets, Several complex variables and complex geometry, Part 2 (Santa Cruz, CA, 1989), 477-508, Proc. Sympos. Pure Math. 52, Amer. Math. Soc., Providence, R.I., 1991.

[Sh] M. ShIRosAKI, An extension of unicity theorem for meromorphic functions, Tohoku Math. J. (2) 45 (1993), 491-497.

[Tu] Z.-H. TU, Uniquenness problem of meromorphic mappings in several complex variables for moving targets, Tohoku Math. J. (2) 54 (2002), 567-579.

[W] J. T.-Y. WANG, A generalization of Picard's theorem with moving targets, Complex Var. Theory Appl. 44 (2001), 39-45.

[Ye] Z. YE, A unicity theorem for meromorphic mappings, Houston J. Math. 24 (1998), 519-531.

DEPARTMENT OF MATHEMATICS FUDAN UNIVERSITY

SHANGHAI

P.R. CHINA

E-mail address: jinluk@online.sh.cn
DEPARTMENT OF MATHEMATICS

UNIVERSITY OF HOUSTON

HOUSTON, TX 77204

USA

E-mail address: minru@math.uh.edu 